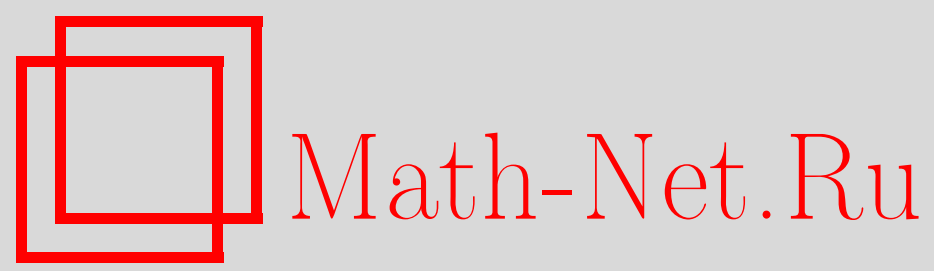

Я. М. Клебанов, И. Е. Адеянов, А. Н. Давыдов, Модель связанных процессов неустановившейся ползучести, теплопроводности и повреждаемости, Beстн. Сам. гос. техн. ун-та. Сер. Физ.-мат. науки, 2003, выпуск 19, 64-69

DOI: https://doi.org/10.14498/vsgtu142

Использование Общероссийского математического портала Math-Net.Ru подразумевает, что вы прочитали и согласны с пользовательским соглашением

http://www.mathnet.ru/rus/agreement

Параметры загрузки:

IP: 54.237 .206 .68

26 апреля 2023 г., 18:23:47 


\section{МОДЕЛЬ СВЯЗАННЫХ ПРОЦЕССОВ НЕУСТАНОВИВШЕЙСЯ ПОЛЗУЧЕСТИ, ТЕПЛОПРОВОДНОСТИ И ПОВРЕЖДАЕМОСТИ}

Рассмотрены новые определяющие уравнения нелинейной вязкоупругости с временной аналогией. Они основаны на термодинамическом анализе связанных прочессов деформирования, повреждаемости и теплопроводности в материале. Найденные зависимости допускают использование любого кинетического закона накопления повреждаемости. В качестве приложения подробно рассматривается деформирование и повреждаемость в полимерах при ичиклическом нагружении. Вводится кинетическое уравнение усталостной повреждаемости полимерных материалов при крэйзинге. Предложенные уравнения проверяются на базе экспериментальных данных процессов ииклической ползучести полиэтилена низкой плотности. Сравнение теоретических результатов с экспериментальными данными показывает, что предложенная модель дает хороший прогноз ускорения проичесса ползучести при ичиклическом нагружении.

Введение. Систематическое развитие современной теории вязкоупругости основывается на законах необратимой термодинамики $[1,2]$. Этот подход также позволяет моделировать процесс развития повреждаемости в вязкоупругих средах [1-3]. Частные законы накопления повреждаемости были введены, опираясь на линейную теорию для неповрежденных вязкоупругих материалов. Накопление повреждаемости в нелинейной вязкоупругой среде было рассмотрено с использованием временной аналогии [4]. Подобный подход может быть применен лишь для описания изотропного накопления повреждаемости. Существуют несколько моделей накопления повреждаемости, связанных с уравнениями нелинейной вязкоупругости, которые были предложены вне рамок термодинамики $[3,4]$.

Основная цель данной статьи - построение определяющих уравнений нелинейной вязкоупругости, учитывающих накопление повреждаемости. С этой целью выполняется термодинамический анализ связанных процессов деформирования, повреждаемости и теплопроводности. Концепция эффективного напряжения в поврежденном материале [5,6] развивается путём введение такой эффективной деформации, на которой эффективное напряжение совершает работу. Показано, что предложенная модель хорошо описывает эффекты циклического нагружения в полимерных материалах.

Определяющие уравнения. Запишем первый и второй законы термодинамики в дифференциальной форме:

$$
\begin{gathered}
\rho d e=d t\left(\rho r-q_{i, i}\right)+\sigma_{i j} d \varepsilon_{i j} ; \\
\rho T d s=d t\left(\rho r-q_{i, i}\right)+\gamma d \xi,
\end{gathered}
$$

где $\rho$ - плотность материала; $t$ - физическое время; $e$ - плотность внутренней энергии; $\sigma_{i j}$ - тензор напряжений; $\varepsilon_{i j}$ - тензор деформаций; $r$ - мощность внешних тепловых источников в единице объёма; $q_{i}$ - вектор теплового потока; $T$ - температура; $s$ - плотность энтропии; $\gamma$ - скорость изменения плотности энергии диссипации, рассматриваемая на шкале приведённого времени.

Скорость изменения приведённого времени определяется соотношением

$$
\frac{d \xi}{d t}=f\left(\varphi_{r}, t\right), r=1,2, \ldots,
$$

где $f$ - неотрицательный масштабный фактор, зависящий от параметров $\varphi_{r}$, в качестве которых могут выступать эквивалентные напряжения, температура, влажность, концентрация пластификатора [7,8], поврежденность, плотность потока нейтронов [9] и т.д. Если $\partial g / \partial t=0$, то приведенное время удовлетворяет условию инвариантности свойств относительно точки нулевого отсчёта времени. В противном случае мы имеем дело со стареющим материалом.

Введя плотность свободной энергии $\psi=e-T s$, из (1) и (2) получаем

$$
\begin{gathered}
\rho d \psi+\rho s d T+\gamma d \xi=\sigma_{i j} d \varepsilon_{i j} ; \\
\rho T \frac{d s}{d t}=\rho r-q_{i, i}+\gamma \frac{d \xi}{d t} .
\end{gathered}
$$

Эффективное напряжение $s_{i j}$ вводится как [6] 


$$
\sigma_{i j}=\left(I_{i j k l}-\mathrm{X}_{i j k l}\right) s_{k l},
$$

где $I_{i j k l}$ - единичный тензор четвертого порядка; $\mathrm{X}_{i j k l}$ - несимметричный тензор повреждаемости.

Также вводится эффективная деформация $e_{i j}$, на которой эффективное напряжение совершает работу:

$$
d e_{k l}=\left(I_{i j k l}-\mathrm{X}_{i j k l}\right) d \varepsilon_{i j}, e_{i j}=\varepsilon_{i j}=0, \text { при at } t=0 .
$$

Так как $\varepsilon_{i j}=\varepsilon_{i j}^{\prime}+\alpha_{i j} \Delta T$, где $\alpha_{i j}$ - коэффициенты температурного расширения, а $\Delta T=T-T_{o}, T_{o}$ начальная температура, то имеем

где

$$
\begin{gathered}
d e_{k l}=d e_{k l}^{\prime}+\left(I_{i j k l}-\mathrm{X}_{i j k l}\right) \alpha_{i j} d T, \\
d e_{k l}^{\prime}=\left(I_{i j k l}-\mathrm{X}_{i j k l}\right) d \varepsilon_{i j}^{\prime} .
\end{gathered}
$$

Равенство (4) теперь может быть записано в виде

$$
\rho d \psi+\rho s d T+\gamma d \xi=s_{i j} d e_{i j} .
$$

Введем свободную энергию в следующей форме:

$$
\psi=\psi_{o}(T)+\frac{1}{2} \int_{0}^{\xi} \int_{0}^{\xi} G_{i j k l}\left(\xi-\varsigma_{1}, \xi-\varsigma_{2}\right) d e_{i j}^{\prime}\left(\varsigma_{1}\right) d e_{k l}^{\prime}\left(\varsigma_{2}\right),
$$

где $\psi_{o}$ - величина, зависящая только от температуры, а ядро обладает свойствами симметрии:

$$
G_{i j k l}\left(\xi_{1}, \xi_{2}\right)=G_{i j k l}\left(\xi_{2}, \xi_{1}\right)=G_{k l i j}\left(\xi_{1}, \xi_{2}\right)=G_{j i k l}\left(\xi_{1}, \xi_{2}\right),
$$

a $G_{i j k l}\left(\xi_{1}, \xi_{2}\right)=0$ при $\xi_{l}<0, \xi_{2}<0$.

Полный дифференциал свободной энергии может быть представлен в виде $d \psi=d T \frac{d \psi_{o}}{d T}+d e_{i j}^{\prime} \int_{0}^{\xi} G_{i j k l}(\xi-\zeta) d e_{k l}^{\prime}(\varsigma)+d \xi \cdot \frac{1}{2} \int_{0}^{\xi} \int_{0}^{\xi} \frac{d}{d \xi} G_{i j k l}\left(\xi-\varsigma_{1}, \xi-\varsigma_{2}\right) d \varepsilon_{i j}^{\prime}\left(\varsigma_{1}\right) d \varepsilon_{k l}^{\prime}\left(\varsigma_{2}\right)$, где $G_{i j k l}(\xi)=G_{i j k l}(\xi, 0)$.

Теперь (8) может быть переписано в виде

$$
\begin{aligned}
d T \rho \frac{d \psi_{o}}{d T}- & d T \rho\left(I_{m n i j}-\mathrm{X}_{m n i j}\right) \alpha_{m n} \int_{0}^{\xi} G_{i j k l}(\xi-\zeta) d e_{k l}^{\prime}(\zeta)+\rho s d T+d e_{i j} \rho \int_{0}^{\zeta} G_{i j k l}(\xi-\zeta) d e_{k l}^{\prime}(\zeta)+ \\
& +d \xi \cdot \frac{1}{2} \rho \int_{0}^{\xi} \int_{0}^{\xi} \frac{d}{d \xi} G_{i j k l}\left(\xi-\zeta_{1}, \xi-\zeta_{2}\right) d e_{k l}^{\prime}\left(\zeta_{1}\right) d e_{i j}^{\prime}\left(\zeta_{2}\right)+\gamma d \xi=s_{i j} d e_{i j} .
\end{aligned}
$$

Приравнивая коэффициенты при независимых приращениях $d T, d e_{i j}$ и $d \xi$ в обеих частях последнего выражения, получаем

$$
\begin{gathered}
\frac{d \psi_{o}}{d T}-\left(I_{m n i j}-\mathrm{X}_{m n i j}\right) \alpha_{m n} \int_{0}^{\xi} G_{i j k l}(\xi-\zeta) d e_{k l}^{\prime}(\zeta)+s=0 ; \\
s_{i j}=\rho \int_{0}^{\xi} G_{i j k l}(\xi-\zeta) d e_{k l}^{\prime}(\zeta) ; \\
\gamma=-\frac{1}{2} \rho \int_{0}^{\xi} \int_{0}^{\xi} \frac{d}{d \xi} G_{i j k l}\left(\xi-\zeta_{1}, \xi-\zeta_{2}\right) d e_{k l}^{\prime}\left(\zeta_{1}\right) d e_{i j}^{\prime}\left(\zeta_{2}\right) .
\end{gathered}
$$

Из (6), (9) и (10) следует, что

$$
\rho \frac{d \psi_{o}}{d T}-\sigma_{m n} \alpha_{m n}+\rho s=0
$$

и

$$
e_{i j}^{\prime}=\frac{1}{\rho} \int_{0}^{\xi} J_{i j k l}(\xi-\zeta) d s_{k l}(\zeta),
$$

где $J_{i j k l}-$ резольвентное ядро. Вводя $C_{\sigma}=T \frac{\partial s}{\partial T}$ при $\sigma_{i j}=c o n s t$, получаем из (11)

$$
\frac{d^{2} \psi_{o}}{d T^{2}}=-\frac{C_{\sigma}}{T} .
$$

Дифференцируя (11) по $t$, находим

$$
\rho T \frac{d s}{d t}=\rho C_{\sigma} \frac{d T}{d t}+T \alpha_{i j} \frac{d \sigma_{i j}}{d t} .
$$


Подставляя (13) в (5) и учитывая закон Фурье $q_{i}=-\lambda_{i j} T,{ }_{j}$, где $\lambda_{i j}$-тензор коэффициентов теплопроводности, получаем полное уравнение

$$
\rho C_{\sigma} \frac{d T}{d t}=\rho r+\left(\lambda_{i j} T,{ }_{j}\right),_{i}+\gamma \frac{d \xi}{d t}-T \alpha_{i j} \frac{d \sigma_{i j}}{d t} .
$$

В случае изотропной повреждаемости вместо тензора $\mathrm{X}_{i j k l}$ вводится скалярная переменная $\mathrm{X}$. Тогда из (7) получаем

$$
\frac{d \varepsilon_{i j}^{\prime}}{d \xi_{1}}=\frac{d e_{i j}^{\prime}}{d \xi}
$$

где $d \xi_{1}=\frac{d \xi}{1-\mathrm{X}}$.

Так как $e_{i j}^{\prime}=\varepsilon_{i j}^{\prime}=0$, при $t=0$, то мы можем переписать (12) в следующем виде:

$$
\varepsilon_{i j}^{\prime}=\frac{1}{\rho} \int_{0}^{\xi_{1}} J_{i j k l}\left(\xi_{1}-\zeta\right) d s_{k l}(\zeta)
$$

Зависимости, полученные в этом разделе, удовлетворяют любой частной модели накопления повреждаемости.

Циклическое нагружение полимеров. Циклическое нагружение полимерных материалов является достаточно актуальным вопросом. Поведение полимеров при ползучести оказывается чувствительным к циклическому характеру нагружения - последнее является причиной значительного ускорения процесса накопления деформации ползучести. Это явление было названо эффектом виброползучести. Оно было отмечено при испытаниях различных полимерных материалов [10-17]. В то же время оказалось, что ползучесть полимеров при периодических внешних воздействиях - это важный фактор, влияющий на число циклов до разрушения [18]. Циклический характер нагружения может также явиться причиной саморазогрева материала в связи с диссипацией энергии.

Важная особенность процесса повреждаемости в полимерных материалах при циклическом нагружении - это скачкообразное возрастание числа дефектов на начальной стадии с последующим замедлением процесса. При этом плотность дефектов - крэйзов с течением времени постепенно приближается к насыщению $[19,20]$. Затем наступает стадия, которая характеризуется стремительным нарастанием дефектов и образованием микротрещин.

Введем кинетическое уравнение усталостной повреждаемости, описывающее подобное поведение. Одноосная модель может быть записана в виде

$$
\delta \mathrm{X}=\frac{1}{\left[1-(1-\mathrm{X})^{v+1}\right]^{\mu}}\left(\frac{F\left(\sigma_{a}, \sigma_{m}, T\right)}{1-\mathrm{X}}\right)^{v} \delta N,
$$

где $N$ - текущее число циклов; $F$ - функция амплитуды напряжения $\sigma_{a}$, среднего напряжения $\sigma_{m}$ и температуры; $v$ - константа; степень $\mu$ в общем случае зависит от нагрузки. Обе последние величины положительны.

Подобная модель введена в [6], но с другим знаком параметра $\mu .$. Положительное значение этого параметра в (15) важно для описания процесса повреждаемости, характер которого приведён выше, так как зависимость (15) слабо сингулярная: $\frac{\partial \mathrm{X}}{\partial N} \rightarrow \infty$ при $N \rightarrow 0$.

Из (15) следует, что при установившемся циклическом нагружении

$$
N_{F}=\frac{1}{(v+1)(\mu+1)\left[F\left(\sigma_{a}, \sigma_{m}, T\right)\right]^{\nu}}, \quad \mathrm{X}=1-\left[1-\left(\frac{N}{N_{F}}\right)^{\frac{1}{1+\mu}}\right]^{\frac{1}{1+v}},
$$

где $N_{F}$ - число циклов до разрушения.

Если повреждаемость рассматривается как аргумент для функции (3), то это усложняет экспериментальное нахождение параметров определяющих уравнений и в каждом конкретном случае должно быть обосновано лучшим соответствием экспериментальным данным. Если учёт влияния повреждаемости на функцию масштабного фактора является целесообразным, то более удобно использовать функцию, зависящую от инварианта тензора эффективного напряжения, чем учитывать повреждаемость как независимый аргумент. Это может быть записано в виде 


$$
\frac{d \xi}{d t}=f\left(s_{e}, T\right)
$$

где $s_{e}$ - эквивалентное эффективное напряжение, однородная функция первого порядка от $\sigma_{i j}$.

Процедура нахождения параметров и функций определяющего уравнения включает несколько шагов. Первая и вторая стадии кривых стационарной ползучести при различных температурах должны быть сначала обработаны с целью введения функций $f$ и $J_{i j k l}$. Если воздействие отдельных параметров (3) на ползучесть является независимым, то масштабный фактор может быть представлен как произведение индивидуальных масштабных факторов, каждый их которых связан со своим собственным параметром. В общем случае, если несколько параметров изменяются одновременно, то введённая зависимость описывает их взаимодействие. Структура функции $f$ и ее константы определяются с помощью методов, описанных в [8].

Кривые ползучести при постоянном напряжении для полиэтилена низкой плотности при $20^{\circ} \mathrm{C}$ показаны на рис. 1 . Они были аппроксимированы следующими функциями и константами:

$$
\begin{gathered}
\rho J_{1111}(\xi)=\frac{1}{E}+\sum_{\tau} a_{\tau}\left(1-e^{-b_{\tau} \xi}\right) ; f\left(\sigma_{11}, T\right)=f_{1}\left(\sigma_{11}\right) f_{2}(T) ; \\
f_{1}\left(\sigma_{11}\right)=0.031+4.22 \cdot 10^{-5} e^{2.83 \sigma_{11}} ; f_{2}(T)=\exp \left(\frac{43.45(T-293)}{129.45+(T-293)}\right),
\end{gathered}
$$

где используется зависимость для $f_{2}(T)$, полученная в [4]. Величины констант материала имеют следующую размерность - МПа $\left(\sigma_{11} E\right),{ }^{\circ} \mathrm{K}(T), \% / \mathrm{MПа}\left(a_{\tau}\right)$, ч $^{-1}\left(b_{\tau}\right)$ и ч $(\xi)$. Часть из них приведена в табл.1.

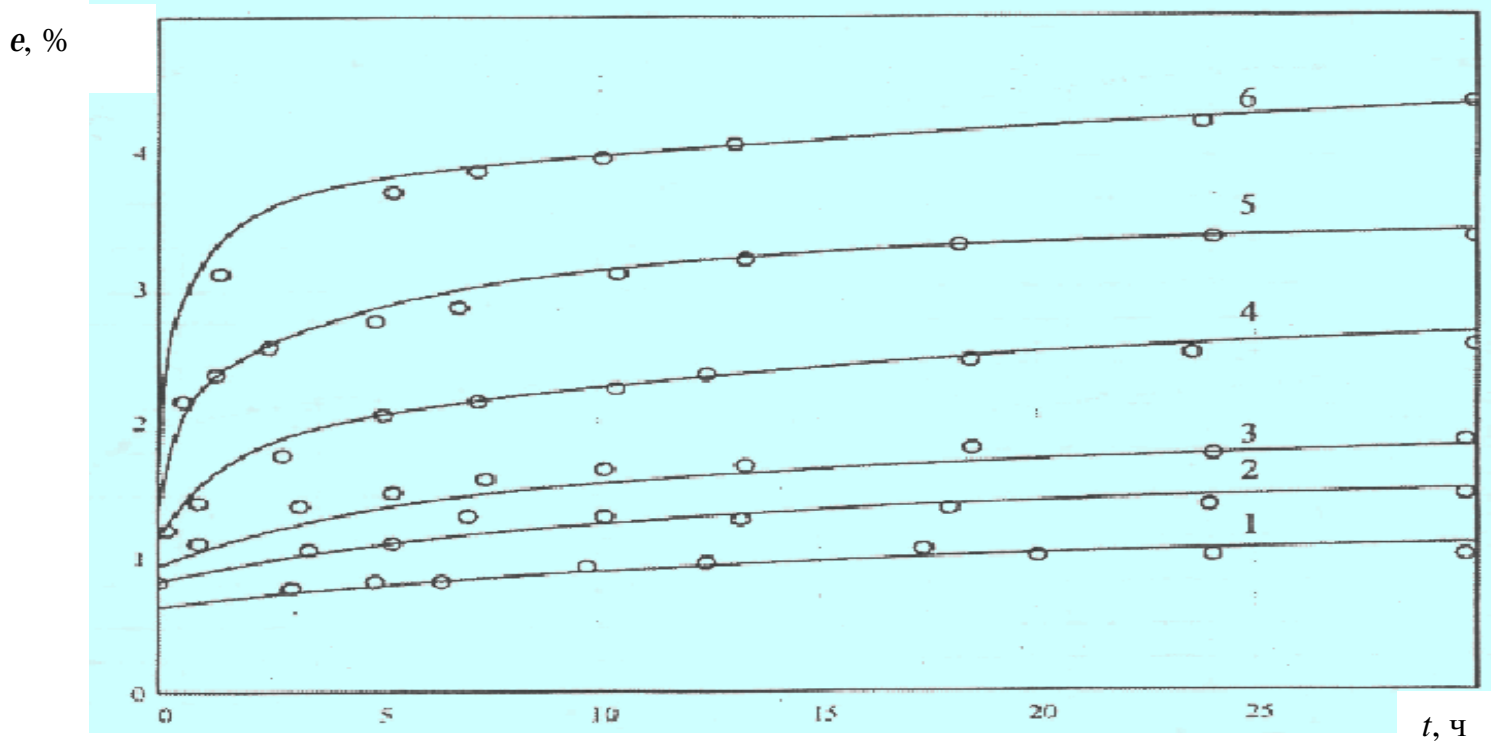

Р и с.1. Диаграмма ползучести полиэтилена низкой плотности при постоянных напряжениях: 1,69 МПа (1); 2,20 МПа (2); 2,51 МПа (3); 3,07 МПа (4); 3,55 МПа (5) и 4,06 МПа (6)

- теоретический прогноз; О О О О 0 - экспериментальные данные

Т а б л и ц а 1

Константы определяющих уравнений для полиэтиле на низкой плотности

\begin{tabular}{|c|c|c|c|c|c|}
\hline \multirow{2}{*}{ Е } & $a_{1}$ & $a_{2}$ & $a_{3}$ & $a_{4}$ & $a_{5}$ \\
& $b_{1}$ & $-b_{2}$ & $-b_{3}$ & $b_{4}$ & $b_{5}$ \\
\hline МПа & \multicolumn{7}{|c|}{$\% / \mathrm{M \Pi a} ; \mathrm{q}^{-1}$} \\
\hline \multirow{2}{*}{270} & 0.218 & 0.231 & 0.098 & 0.248 & 0.277 \\
& 2.6 & 0.26 & $-\ldots .1$ & -2.6 & 0.0006 \\
\hline
\end{tabular}


Циклические испытания [4] были проведены при одном и том же среднем напряжении 3,57 МПа, различных амплитудах напряжения и частотах, представленных в табл.2.

Т а б л и ц а 2

\section{Условия циклического нагружения в испытаниях полиэтилена низкой плотности}

\begin{tabular}{|c|c|c|c|c|}
\hline \multirow{2}{*}{ Программа } & \multirow{2}{*}{$\begin{array}{c}\text { Частота, } \\
\text { Гц }\end{array}$} & $\begin{array}{c}\text { Амплитуда напря- } \\
\text { жений, } \\
\text { МПа }\end{array}$ & \multicolumn{2}{|c|}{ Установившаяся температура, ${ }^{\circ} \mathrm{K}$} \\
\cline { 3 - 5 } & 150 & 0.59 & 305 & Эеоретическая \\
\hline $\mathrm{a}$ & 250 & 0.39 & 301.5 & 305.0 \\
\hline $\mathrm{b}$ & 250 & 0.45 & 304.5 & 301.6 \\
\hline $\mathrm{c}$ & 250 & 0.49 & 302 & 307.0 \\
\hline $\mathrm{d}$ & 400 & 0.32 & 308 & 302.1 \\
\hline $\mathrm{e}$ & 300 & 0.43 & 305 & 305.9 \\
\hline $\mathrm{f}$ & 250 & 0.53 & 312 & 315.2 \\
\hline $\mathrm{g}$ & 250 & 0.62 & 315 & \\
\hline $\mathrm{h}$ & & & & \\
\hline
\end{tabular}

Влияние последнего члена в уравнении (14) незначительно. Принимая далее отсутствие внешнего подвода тепла и вводя усреднённую температуру за один цикл $T^{c}$ [21], приходим к следующему результату:

$$
\rho C_{\sigma} \frac{d T^{c}}{d t}=\left(\lambda_{i j} T,{ }_{j}\right)_{, i}+\gamma_{c},
$$

где $\gamma_{c}$ - усредненная за цикл скорость диссипации. При изотропных и однородных свойствах теплопроводности $\lambda_{i j}=\delta_{i j} \lambda$ и $\left(\lambda_{i j} T,{ }_{j}\right),_{i}=\lambda T,_{i i}$.

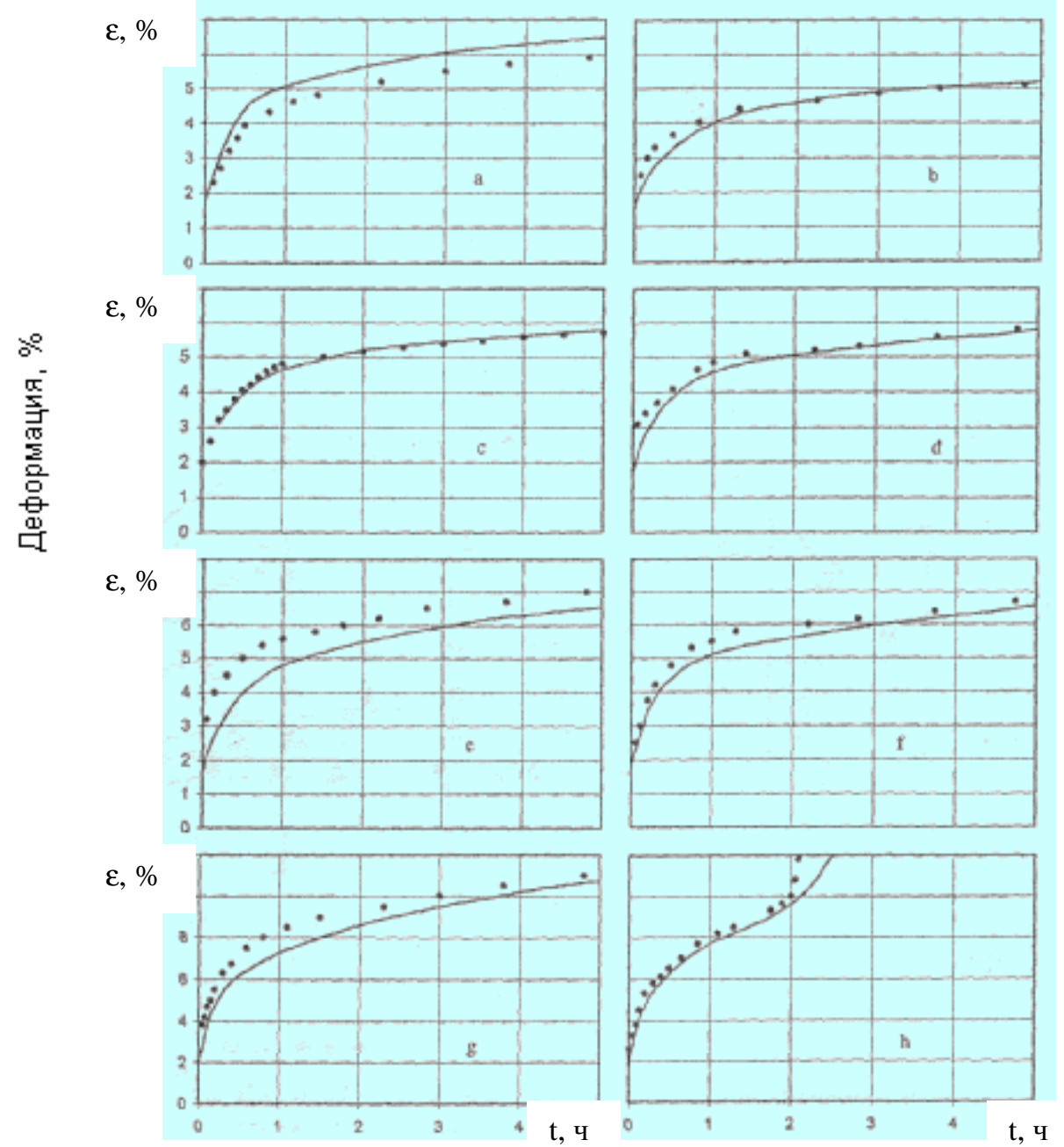

Р и с. 2. Виброползучесть полиэтилена низкои плотности. Режимы нагружения даны в табл. 2: - теоретический прогноз;0 0 0 0 о - экспериментальные данные 
Число циклов до разрушения было аппроксимировано зависимостью

$$
N_{F}=\frac{1}{(v+1)(\mu+1) f_{2}(T)}\left(\frac{\sigma_{a}}{C}\right)^{-v},
$$

где $v=5, \mu=23.5, C=102.8$ МПа. Произведение $N_{F} f_{2}(T)$ не зависит от температуры и может рассматриваться как число циклов до разрушения на шкале приведенного времени.

Для определения параметров $\mu$ и $C$ мы использовали данные циклической ползучести для программ " $b$ " и “ $g$ " при $t=5$ ч, а для определения параметра $v$ также был использован экспериментально найденный график зависимости числа циклов до разрушения [18]. Теоретические результаты были получены при коэффициенте Пуассона, равном 0,5 [22]. Коэффициент термического расширения для полиэтилена низкой плотности равен $1 \cdot 10^{-4} 1 /{ }^{\circ} \mathrm{K}[23]$; другие харак-

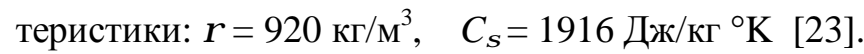

Влияние повреждаемости на масштабный фактор при циклическом нагружении учитывается формулой (16). Экспериментальные и теоретические кривые циклической ползучести сравниваются на рис. 2. Совпадение кривых вполне удовлетворительное.

\section{БИБЛИОГРАФИЧЕСКИЙ СПИСОК}

1. Christensen R.M. Theory of Viscoelasticity: An Introduction. New York \& London: Academic Press, 1971. 245 p.

2. Илюшин А.А., Победря Б.Е. Основы математической теории термовязкоупругости. М.: Наука, 1970. 280 с.

3. Ахундов М.Б. Повреждаемость и деформирование нелинейных наследственных сред при сложнонапряженном состоянии. // Механика композитных материалов. № 2. С. 235-239.

4. Уржумиев Ю.С., Максимов Р.Д. Описание деформационных свойств полимерных материалов. Рига: Zinatne, $1975.416 \mathrm{c}$.

5. Качанов Л.M. Introduction to Continuum Damage Mechanics. Dordricht: Martinus Hijhoff, 1986. 135 p.

6. Lemaitre J., Chaboche J.-L. Mechanics of Solid Materials. Cambridge: University Press, 1990. 556 p.

7. Бугаков И.И., Шеповецкий М.А. Сравнительное исследование нелинейных уравнений вязкоупругости // Тр. Акад. наук Арм. ССР. Механика. 1984. № 1. С. 56-63.

8. Уржумиев Ю.С. Прогнозирование длительной прочности полимерных материалов. М.: Наука, 1982. $221 \mathrm{c}$.

9. Клебанов Я.М., Самарин Ю.П. Generalized Structural models of stresses in finite element analysis for creep // Proceeding of the 3rd ACME Conference on Computational Mechanics in the UK. University of Oxford. 1995. P. 81-84.

10. Askadskii A.A., Matveev Yu.I., Nurmulhametov F.H., Slonimski G.L.i, Tartakovskii B.D.. Interference of stress and vibration relaxation processes in the sound frequency range. // Polymer Mechanics (Mekhanika Polimerov). 1975. №. 2. P. $287-293$.

11. Bartenev G.M., Shelkovnikova L.A.. Vibrorelaxation of stresses in rubbers. // Polymer Mechanics (Mekhanika Polimerov) 1972. № 4. P. 623 - 625.

12. Fitzgerald J.J., Martellock A.C., Nielsen P.L., Schillace R.V. The Effect of Cyclic Stress on the Physical Properties of a Poly(Dimethylsiloxane) Elastomer // Polymer Engineering and Science. 1992. № 18. P. 1350 - 1357.

13. Lesser A.J. Effective Volume Changes During Fatigue and Fracture of Polyacetal // Polymer Engineering and Science. 1996. № 18. P. 2366-2374.

14. McKenna G.B., Zapas L.J. Response of carbon black filled butyl rubber to cyclic loading // Rubber Chemistry and Technology. 1981. № 54. P. 718 - 733.

15. Nurmulhametov F.H., Dashevskii V.G., Askadskii A.A., Slonimskii G.L. A study of the effect of vibration in the sonic frequency region on the stress-relaxation process in polymers // Polymer Mechanics (Mekhanika Polimerov). 1976. № 4. P. $519-529$.

16. Slonimskii G.L., Alekseev P. A study of vibration influence on relaxation processes in rubbers // Doklady Akademii Nauk SSSR (Transaction of the Science Academy of the USSR). 1956. № 6. P. $1053-1056$.

17. Xieng H. Damage Theory for Polmeric Material // Applied Mathematics and Mechanics. 1999. № 3. P. 338-342.

18. Hertzberg R.W., Manson J.A.. 1980. Fatigue of Engineering Plastics. New York: Academic Press, 295 p.

19. Argon A.S., Hankoosh J.G. Initiation of crazaes in polysterene // Philosophical Magazine. 1977. № 36. P. 1195-1216.

20. Jie M., Tang C.Y., Li Y.P., Li C.C.. Damage evolution and energy dissipation of polymers with crazes // Theoretical and Applied Fracture Mechanics. 1998. № 3. P. 165-174.

21. Molinary A., German Y. Self heating and thermal failure of polymers sustaining a compressive cyclic loading // International Journal of Solids and Structures. 1996. № 23. P. 3439-3462.

22. Masia L. Thermoplastics Materials Engineering. Applied Science publishers: Amsteredam, London, NY. 1989. 446 p.

23. Derham C.J., Tomas A.G. Creep of rubber under repeated stressing // Rubber Chemistry and Technology. 1977. № 50. P. $397-402$. 